\title{
Recurrence after GnRHa treatment vs. observation in women with multiple leiomyomas after myomectomy: study protocol for a multicenter, prospective, randomized controlled clinical trial
}

Jia Wei

Tongji Hospital of Tongji Medical College of Huazhong University of Science and Technology

Xiangyi Ma

Tongji Hospital of Tongji Medical College of Huazhong University of Science and Technology

Wenwen Wang

Tongji Hospital of Tongji Medical College of Huazhong University of Science and Technology

Minli Zhang

Tongji Hospital of Tongji Medical College of Huazhong University of Science and Technology zhiying Yu

Shenzhen University

Wei Zhang

Wuhan University Zhongnan Hospital

Li Hong

Wuhan University Renmin Hospital

zhiyin Li

Three Gorges University Renhe Hospital

Lin Li

Xiangyang Central Hospital

\section{Yan Wang}

Huazhong University of Science and Technology

\section{Yun Feng}

First People's Hospital of Yunnan

Ruixia Guo

Zhengzhou University

Chunlian Zhang

Taihe Hospital

\section{Wuliang Wang}

Zhengzhou University

\section{Qingfen Yue}

Zhengzhou University 
Shixuan Wang ( $\nabla$ sxwang@tjh.tjmu.edu.cn )

Huazhong University of Science and Technology Tongji Medical College

\section{Study protocol}

Keywords: Multiple leiomyomas, GnRHa, Recurrence, Randomized controlled trial

Posted Date: August 12th, 2019

DOI: https://doi.org/10.21203/rs.2.12625/v1

License: (c) (1) This work is licensed under a Creative Commons Attribution 4.0 International License. Read Full License 


\section{Abstract}

Background: Recurrence of the leiomyoma remains as one of the major concerns of myomectomy in the long-term management, especially for the multiple leiomyomas. Gonadotropin-releasing hormone agonist ( $\mathrm{GnRHa}$ ) is currently one of the most effective medications to reduce the volume of the fibroids and the uterus. However, its role in preventing recurrence after conservative surgery remains unclear. At present, there is no evidence from randomized clinical trials comparing the efficacy between GnRHa and follow-up observation in the recurrence rate of multiple leiomyomas after myomectomy. Methods/design: We are conducting a randomized controlled trial in women aged 18-45 undergoing myomectomy for the multiple leiomyomas. After the surgery, women whose pathological result confirms leiomyoma are randomized into two parallel groups: observation group and GnRHa group. The primary outcome is recurrence of either clinical symptoms or imaging. Discussion: The results of this study will provide evidence for the efficacy of the gonadotropin-releasing hormone agonist in preventing the recurrence of high-risk leiomyoma after myomectomy.

\section{Background}

Leiomyoma, which is also called myoma or uterine fibroid, is the most common benign uterine tumor among women of reproductive age and its clinical presentations include pelvic mass, chronic pelvic pain, abnormal uterine bleeding and infertility (Donnez \& Jadoul, 2002). Leiomyoma occurs in $50-60 \%$ of women of reproductive age, rising to $70 \%$ by the age of 50 (Baird, Dunson, Hill, Cousins, \& Schectman, 2003), which translates into 880 million women worldwide. It originates from the smooth muscle cells in the myometrium and highly dependent on estrogen stimulation. Multiple uterine fibroids are one of the special forms of leiomyoma, as it refers to multiple myomas which can be subendometrial, intramural, subserosal, and combinations of these. It is of high risk of recurrence (Fauconnier, Chapron, Babaki-Fard, \& Dubuisson, 2000; Radosa et al., 2014).

Currently the treatment for multiple leiomyomas involve mainly surgical interventions. However, the approach of surgery is largely dependent on the patients' age and will to preserve the fertility. The commonly accepted concept for surgical interventions now is to avoid "radical" surgery such as hysterectomy in order to prevent pelvic organs prolapse. Therefore, myomectomy by either hysteroscopy or laparoscopy has become the dominant choice. However, it is widely known that the recurrence rate for leiomyoma after myomectomy can be as high as $60 \%$ after 5 years (Donnez, Donnez, \& Dolmans, 2014; MALONE, 1969). As for multiple leiomyomas the recurrence is even higher (MALONE, 1969).

Since the growth of leiomyoma is heavily influenced by estrogen level, gonadotropin-releasing hormone agonist $(\mathrm{GnRHa}$ ) was first investigated as a potential treatment since 1980s. It is confirmed that $\mathrm{GnRHa}$ can decrease the volume of fibroid and uterus (Falsetti, Mazzani, Rubessa, \& Ruggeri, 1992; Jasonni et al., 2001). Currently GnRHa has been used pre-operationally as it can decrease the pre-operative and perioperative blood loss therefore improves the condition of the surgery (Zhang et al., 2014). However, 
there is lack of clinical trials looking into the agents of preventing recurrence of the multiple leiomyomas after conservative surgery..

At present, there is no guideline for the long term management in multiple leiomyomas. A randomized trial is warranted to test the efficacy of the $\mathrm{GnRHa}$ in the prevention of the recurrence of the multiple leiomyoma. This study is a multicenter randomized controlled trial comparing the efficacy of GnRHa with observation in recurrence prevention among women had myomectomy.

\section{Methods}

\section{Study population}

This study is conducted in 12 acedemic hospital locatated in central China.

\section{Inclusion criteria}

The inclusion criteria are as follows:

- Women aged $\geq 18$ and $\leq 45$ years

- Women who are diagnosed with multiple leiomyomas clinically

- Women who undergo myomectomy and are confirmed leiomyoma by pathology lab

- Ultrasound confirms no fibroid or the diameter smaller than $2 \mathrm{~cm}$ after the surgery

- Women who don't used medications for leiomyoma 3 months before the enrollment

- Women who can give written consent

\section{Exclusion criteria}

The exclusion criteria are as follows:

- Women who have been diagnosed with congenital uterine abnormalities such as uterine malformation (uterus unicornis, septate uterus, or duplex uterus)

- Women who are pregnant

- Women who are diagnosed with other estrogen dependent diseases such as adenomyoma or endometriosis

- Women with medical conditions that contraindicate surgery, such as uncontrolled hypertension or known symptomatic heart disease; poorly controlled type 1 or type 2 diabetes mellitus; undiagnosed liver disease or dysfunction (based on serum liver enzyme test results); renal disease or abnormal serum renal function; severe anemia; history of deep venous thrombosis, pulmonary embolus, or cerebrovascular accident; history of (or suspected) cervical carcinoma, endometrial carcinoma, or breast carcinoma.

- Women who are allergic to the GnRHa 
- Women who are also undertake other clinical trials at the same time

\section{Study Intervention}

\section{Screening}

At the screening visit, previous medical history and current medication status are reviewed with the standardized case report forms. A physical examination and imaging such as transvaginal ultrasound scan or MRI are performed. Laboratory measurements including basal sex hormone tests, AMH, safety assays such as liver function, renal function, hepatitis virus, HIV, syphilis, coagulation, blood routine, and urine routine are performed in the local labs of the study sites. Quality of life is recorded using the SF-36 and pictorial blood loss assessment chart, PBAC.

\section{Myomectomy}

Myomectomy by either laparoscopy or open surgery is performed in patients who are suspected to have multiple leiomyomas. Myomectomy can be conducted by laparoscopic, hysteroscopic or abdominal approach. The length of the surgery, numbers and size of the fibroids, blood loss and the integrity of the uterine cavity should be kept in record.

\section{Enrollment}

Written informed consent will be obtained from the patients after the pathological lab comfirmed the leiomyomas.

\section{Randomization and safety}

Simple randomization is used to assign subjects to two groups with a 1:1 ratio. The randomization is stratified by study site. The sequence of randomization has been generated by biostatisticians in data coordinator center with Microsoft excel. The original sequence is safely kept by the staff in the data coordinator center, and it has been input into the online central randomization system by these staff members, who are not involved in enrolling subjects. The online sequence is not accessible to any investigators or study coordinators. If a subject fulfills the enrollment criteria, the authorized study coordinator will get the assignment for her. After randomization, both subjects and investigators are informed about the assignments. All potential adverse events will be monitored throughout the trial, with the supervision from the Huazhong University of Technology and Science Ethnic committee. Participants can quit the trial anytime when there is intolerance of the therapy or serve side effects.

\section{The gonadotropin-releasing hormone agonist (GnRHa)}


Participants in the treatment group will receive GnRHa 3.75mg intramuscular injection (Diphereline, Ipsen, France) day one of the menstruation after the surgery for six courses. Hypogonadic side effects caused by medication such as hot flushes, bone loss should be carefully evaluated and could be treated with add-back therapy to maintain estradiol at the level of $30-50 \mathrm{pg} / \mathrm{ml}$.

A schedule of enrollment, interventions, and assessment is provided as in the table below (Table. 1). The flow chart of this study is given in Fig. 1

Figure 1. The flowchart of the study

\section{Follow-up \\ Follow up}

There are seven followups after the surgery, every three months in the first two visits and every six months in the following visits. During each visit, the sex hormones, $\mathrm{AMH}$ is tested and the pelvic ultrasound is prescribed. The SF-36/PBAC is also recorded.

The patients can quit the trial when the side effects of the medication is unbearable or they are not willing to participate. The trial should immediately stop when there is any adverse events. Adverse events are any unfavorable medical occurrences associated with the subject's participation in the research, whether or not considered related to the study intervention.

Serious adverse events are events that are temporally associated with the subject's participation in research that meet any of the following criteria: death, life-threatening, severely or permanently disabling, requiring in-patient hospitalization or prolongation of existing hospitalization, or any events deemed as serious by the local principal investigator.

\section{Outcome And Outcome Assessments}

The primary outcome is recurrence. The secondary outcomes include uterine size, menstruation blood loss, clinical pregnancy, ovarian reserve, quality of life and other adverse events. Regarding recurrence, it defines as the recurrence of symptoms or the growth of fibroids. The symptoms include lower abdominal pain, increased menstruation blood loss and abnormal uterine bleeding. The growth of fibroids is defined as the diameter of the fibroid larger than $2 \mathrm{~cm}$ or the number increased compared to the baseline image after the surgery.

As for menstruation blood loss, it is assessed by pictorial blood loss assessment chart.

\section{Data Analysis}

Sample size calculation 
Based on the retrospective data from our department, the recurrence rate of multiple leiomyomas 3 years after the surgery is about $30 \%$. It was assumed that an absolute difference of $10 \%$ in recurrence rate will be of clinical significance. We aim to test a difference of $10 \%$ of recurrence rate between treatment and observation groups (i.e., $20 \%$ in the $\mathrm{GnRHa}$ group and $30 \%$ in the observation group) at a significance level of 0.05 with a statistical power of $80 \%$. The minimal sample size calculated is 291 for each group. In consideration of a dropout rate of $10 \%$, we will enroll 320 subjects in each group.

\section{Data collection}

Patient will be recruited from March 2018 to March 2020, and follow up will be done three years after the surgery. Data are collected with a standard case report form. Data are de-identified before being input into the database. Regular study site monitor and database checking are performed to ensure the accuracy of data collected.

\section{Data monitoring}

The data monitoring committee (DMC) is consist of trial manager, a third party quality control committee and the head of the department of the central center who is in charge of the progress of the study. This is independent from any funding bodies. The DMC has access to the interim data and can make the decision to terminate the trial when there is advers effect during intervention compare to observation.

\section{Data analysis}

Data analysis and reporting will be conducted in accordance to the CONSORT guideline, which were recorded in our flow chart (Fig 1), including the number of eligible participants and lost to follow-up for various reasons.

Intention to treat will be used as a foundation in our analysis. Comparisons of the characteristics at baseline will be carried out between control and intervention groups. Continuous data will be summarized by means and standard deviation with Wilcoxon rank sum test to identify differences of baseline characteristic between two groups. Categorical data will be described by number and percentages, using Pearson chi-square test to compare discrepancy between groups.

The primary outcome measure is the recurrence rate between two groups after three years' follow-up, which will be analyzed by Pearson chi-square test. For efficacy parameters, such as score of menstruation blood loss, size of uterus and lesion will be analyzed using generalized estimating equations to account for correlations among these observations in different follow up points.

The parameters from secondary outcomes contains SF-36 score, PBAC score are calculated during three years' follow-up are using mixed effects model repeated measures analysis to compare differences 
between two groups at different time points.

The number of participants with adverse events or serious adverse events will be presented for each treatment arm. We will not take any formal statistical testing.

\section{Discussion}

This is a study comparing the efficacy of GnRHa with observation in women who have had myomectomy for multiple leiomyomas. We plan to enroll 640 subjects from 12 teaching hospitals in China. The enrollment began in March 2018.

At the time of manuscript preparation, more than 270 subjects have been enrolled. The result of this large multicenter randomized trial will provide level I evidence for the strategy of long-term management for multiple leiomyomas after myomectomy.

Currently the treatment for leiomyoma is largely rely on surgery, while hysterectomy is more radical it can also cause more complications in short and long term. Myomectomy on the other hand, can preserve the integrity of the uterus ("ACOG practice bulletin. Alternatives to hysterectomy in the management of leiomyomas," 2008) while the recurrence is as high as $30 \%$ three years after the surgery. Second surgery would be more difficult because of the iatrogenic adhesion and sometimes hysterectomy is inevitable. The etiology of leiomyoma is still not fully understood, however epigenetic and metabolic abnormalities are identified in a large percentage of the leiomyoma (Holdsworth-Carson, Zaitseva, Vollenhoven, \& Rogers, 2014; Medikare, Kandukuri, Ananthapur, Deenadayal, \& Nallari, 2011). These could be of potential value for the development of new therapeutic strategies (Commandeur, Styer, \& Teixeira, 2015). Medications for leiomyoma are mostly based on hormone modulation, such as selective progesterone receptor modulators (SPRMs), GnRHa and combined contraception pill (COP). While COP is contradicted in women aged more than 40, the effectiveness of SPRMs versus other treatments is also not clear (Murji, Whitaker, Chow, \& Sobel, 2013). GnRHa was firstly used in treating leiomyoma since 2010 and it has been confirmed that preoperative GnRHa can significantly reduce uterine and fibroid volume (Lethaby, Puscasiu, \& Vollenhoven, 2017). However, there is lack of clinical trial investigating the effect of $\mathrm{GnRHa}$ in preventing the recurrence of leiomyoma after myomectomy.

At present, the long-term management of leiomyoma lacks of a standard guideline. This study is expected to provide a reliable answer to that whether GnRHa can protect recurrence of multiple leiomyomas.

\section{Trial Status}

The enrollment of this study is ongoing at the time of manuscript submission. The trial was registered in Chinese Clinical Trial Registry, ChiCTR-IPR-17012992 (http://www.chictr.org.cn/showproj.aspx?proj = 21797) on 15 Oct 2017. The protocol version number is v.1.0 (Nov-10-2017) and the recuitement date is March-1-2018 and it will take approximately 2 years to complete the recruitment. 


\section{Abbreviations}

COP Combined contraception pill

SPRMs Selective progesterone receptor modulators

PBAC Pictorial blood loss assessment chart

GnRHa Gonadotropin-releasing hormone agonist

\section{Declarations}

All authors declare no compete of interests.

\section{Funding}

This study is a researcher iniciated study and was not funded by any external commercial bodies or internal research grant.

\section{Ethics approval and consent to participate}

The experiments "Multi center, randomized trial for evaluation of the therapeutic effect of GnRHa in reducing the recurrent rate of multiple uterine fibroids post-surgery' involving randomized clinical trial on human were approved by Medical Ethics Committee of the Tongji Hospital Affiliated to Tongji Medical college of Huazhong University of Science and Technology (TJ-IRB20180311) according to submitted study protocol (Version 1.0, 2017-Nov-10) and informed consent (Version 1.0, 2017-Nov-10). The ethical approval was approved by both central and local levels. Central ethical approval has been confirmed from the Medical Ethics Committee of the Tongji Hospital (ref approval no. TJ-IRB20180311) and we will not begin recruiting at other centres in the trial until local ethical approval has been obtained.

\section{Consent for publication}

Not applicable

\section{Confidentiality}

All patient information will be replaced by trial sequence number during the trial and the original data will be kept by the trial corodinators in order to protect the patients confidentiality.

\section{Availability of data and material}




\section{Dissemination policy}

The result of this study will be published in scientific journal.

\section{Authors contributions}

All authors were invlolved in the study design. $\mathrm{J} W$ and $X Y M$ comtrisbute to data analysis and drafting the work. WW W and ML Z were responsible for the data collection and management, ZY Y, W Z, L H, ZY $L, L L, Y W, Y F, R X G, C L Z, Q F Y$ and WL W were in charge for the participants recruitment in different centres, Shixuan Wang was responsible for the study design and revision of the work.

All authors have approved the submitted version of the work.

\section{Acknowledgement}

We thank all clinicians and patients participating in this study.

\section{References}

\section{Reference:}

ACOG practice bulletin. Alternatives to hysterectomy in the management of leiomyomas. (2008). Obstet Gynecol, 112(2 Pt 1), 387-400. doi:10.1097/AOG.0b013e318183fbab

Baird, D. D., Dunson, D. B., Hill, M. C., Cousins, D., \& Schectman, J. M. (2003). High cumulative incidence of uterine leiomyoma in black and white women: ultrasound evidence. Am J Obstet Gynecol, 188(1), 100107.

Commandeur, A. E., Styer, A. K., \& Teixeira, J. M. (2015). Epidemiological and genetic clues for molecular mechanisms involved in uterine leiomyoma development and growth. Hum Reprod Update, 21(5), 593615. doi:10.1093/humupd/dmv030

Donnez, J., Donnez, O., \& Dolmans, M. M. (2014). With the advent of selective progesterone receptor modulators, what is the place of myoma surgery in current practice? Fertil Steril, 102(3), 640-648. doi:10.1016/j.fertnstert.2014.06.041

Donnez, J., \& Jadoul, P. (2002). What are the implications of myomas on fertility? A need for a debate? Hum Reprod, 17(6), 1424-1430. 
Falsetti, L., Mazzani, M. D., Rubessa, S., \& Ruggeri, C. (1992). Presurgical treatment of uterine fibroids by using gonadotropin-releasing hormone agonists. Acta Eur Fertil, 23(1), 29-32.

Fauconnier, A., Chapron, C., Babaki-Fard, K., \& Dubuisson, J. B. (2000). Recurrence of leiomyomata after myomectomy. Hum Reprod Update, 6(6), 595-602.

Holdsworth-Carson, S. J., Zaitseva, M., Vollenhoven, B. J., \& Rogers, P. A. (2014). Clonality of smooth muscle and fibroblast cell populations isolated from human fibroid and myometrial tissues. Mol Hum Reprod, 20(3), 250-259. doi:10.1093/molehr/gat083

Jasonni, V. M., D’Anna, R., Mancuso, A., Caruso, C., Corrado, F., \& Leonardi, I. (2001). Randomized doubleblind study evaluating the efficacy on uterine fibroids shrinkage and on intra-operative blood loss of different length of leuprolide acetate depot treatment before myomectomy. Acta Obstet Gynecol Scand, 80(10), 956-958.

Lethaby, A., Puscasiu, L., \& Vollenhoven, B. (2017). Preoperative medical therapy before surgery for uterine fibroids.

MALONE, L. J. (1969). Myomectomy: Recurrence after Removal of Solitary and Multiple Myomas. 34(2), 200-203.

Medikare, V., Kandukuri, L. R., Ananthapur, V., Deenadayal, M., \& Nallari, P. (2011). The genetic bases of uterine fibroids; a review. J Reprod Infertil, 12(3), 181-191.

Murji, A., Whitaker, L., Chow, T. L., \& Sobel, M. L. J. C. D. o. S. R. (2013). Selective progesterone receptor modulators (SPRMs) for uterine fibroids. 4(10), CD 010770.

Radosa, M. P., Owsianowski, Z., Mothes, A., Weisheit, A., Vorwergk, J., Asskaryar, F. A.,... Runnebaum, I. B. (2014). Long-term risk of fibroid recurrence after laparoscopic myomectomy. Eur J Obstet Gynecol Reprod Biol, 180, 35-39. doi:10.1016/j.ejogrb.2014.05.029

Zhang, Y., Sun, L., Guo, Y., Cheng, J., Wang, Y., Fan, S., \& Duan, H. (2014). The impact of preoperative gonadotropin-releasing hormone agonist treatment on women with uterine fibroids: a meta-analysis. Obstet Gynecol Surv, 69(2), 100-108. doi:10.1097/0GX.0000000000000036

\section{Table}

Table 1. Schedule of enrollment, intervention and assessment 


\begin{tabular}{|c|c|c|c|c|c|c|c|c|c|}
\hline \multirow[t]{2}{*}{ Evaluation } & \multirow[t]{2}{*}{ Screening } & \multirow[t]{2}{*}{ Enrollment } & \multicolumn{4}{|c|}{$\begin{array}{l}\text { Time for } \\
\text { evaluation } \\
\text { after } \\
\text { (months) }\end{array}$} & \multicolumn{3}{|c|}{$\begin{array}{r}\text { follow-up } \\
\text { myomectomy }\end{array}$} \\
\hline & & & 3 & 6 & 12 & 18 & 24 & 30 & 36 \\
\hline Written consent & & $\sqrt{ }$ & & & & & & & \\
\hline Medical history & $\sqrt{ }$ & & & & & & & & \\
\hline Physical exam & $\sqrt{ }$ & & & & & & & & \\
\hline $\begin{array}{l}\text { Safety test (FBC, liver and renal } \\
\text { test, coagulation, ECG) }\end{array}$ & $\sqrt{ }$ & & & & & & & & \\
\hline Ultrasound/MRI & $\sqrt{ }$ & & $\sqrt{ }$ & $\sqrt{ }$ & $\sqrt{ }$ & $\sqrt{ }$ & $\sqrt{ }$ & $\sqrt{ }$ & $\sqrt{ }$ \\
\hline Pathological reslut & & $\sqrt{ }$ & & & & & & & \\
\hline $\mathrm{FSH}, \mathrm{E}_{2}, \mathrm{AMH}$ & $\sqrt{ }$ & & $\sqrt{ }$ & $\sqrt{ }$ & $\sqrt{ }$ & & $\sqrt{ }$ & & $\sqrt{ }$ \\
\hline Side effects & & & $\sqrt{ }$ & $\sqrt{ }$ & $\sqrt{ }$ & $\sqrt{ }$ & $\sqrt{ }$ & $\sqrt{ }$ & $\sqrt{ }$ \\
\hline SF-36/PBAC & $\sqrt{ }$ & & $\sqrt{ }$ & $\sqrt{ }$ & $\sqrt{ }$ & $\sqrt{ }$ & $\sqrt{ }$ & $\sqrt{ }$ & $\sqrt{ }$ \\
\hline
\end{tabular}

Figures 


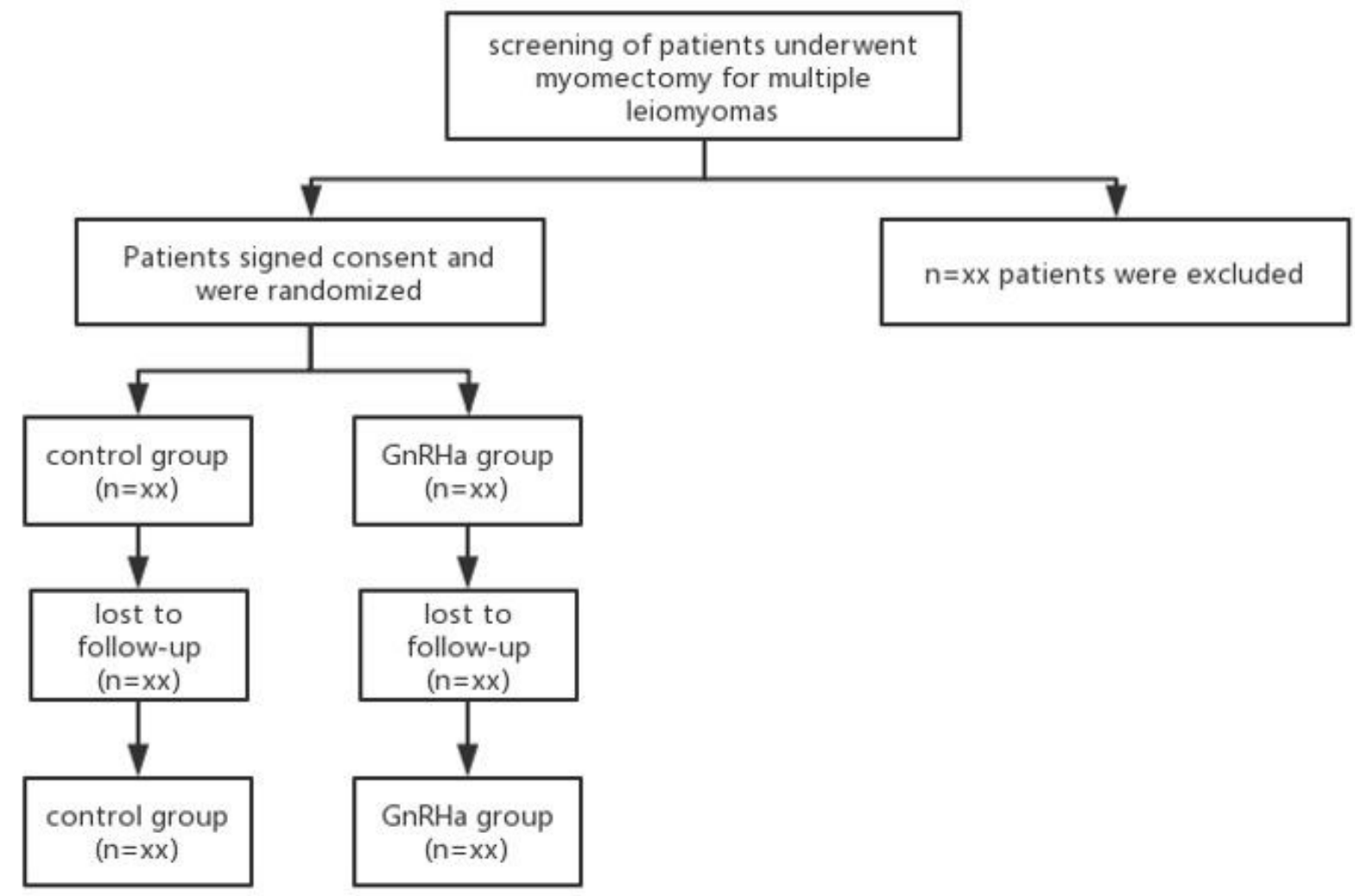

Figure 1

The flowchart of the study

\section{Supplementary Files}

This is a list of supplementary files associated with this preprint. Click to download.

- ethicalapproval.pdf

- checklist.docx

- SPIRITChecklistforrandomisedstudies.doc

- fundingdeclaration.docx 